\title{
Wireless Transmission Based on GPRS Used in Cable On-line Monitoring System
}

\author{
Li Zhou ${ }^{1}$, Yunlong Sun ${ }^{2}$, Jie Chen ${ }^{1, *}$ and Huan Zheng ${ }^{2}$ \\ ${ }^{1}$ Jiangsu Electric Power Research Institute, 211103 Nanjing, P. R. China \\ ${ }^{2}$ Harbin University of Science and Technology, 150080 Harbin, P. R. China \\ ${ }^{*}$ Corresponding author
}

\begin{abstract}
With the Cross Linked Polyethylene (XLPE) power cables increasingly widely used in urban power transmission and distribution network, the reliable operation of XLPE insulated power cable line is becoming more and more important for economic and social development. [1] And there is a need to ensure the reliability of cables by detecting the cable insulation aging state on-line. So that, we developed a set of cable on-line monitoring system which can determine the aging state by the analysis of the current flowing through the cable insulation based on current comparator. Such a distributed measuring system requires the data to be transferred in a wireless way in real time. In order to ensure the reliability of communication, several programs were presented to solve the problem of wireless data transmission between two test equipment based on General Packet Radio Service (GPRS) in this paper. The most suitable one will be select from these programs by analyzing advantages and disadvantages of them to direct the follow-up system debugging.
\end{abstract}

Keywords-cable on-line monitoring; insulation aging state; wireless data transmission; GPRS

\section{INTRODUCTION}

The designed cable insulation test system adopted the method of measuring the current of the cable insulation at the initiation test terminal and the end one. This method is similar to that in reference [2], but the current comparator was used to acquire the current signal instead. By the analysis of these data we can diagnose the aging state of the cable insulation. Remote data synchronization acquisition and reliable data transmission is the basic of the online monitoring system with the self-balanced differential current comparator bridge. When the online monitoring system with the self-balanced differential current comparator bridge is working, the data we get from the double-end system should be transferred in real time. It is the key to ensure that the data transfer steady in high speed and not to be lost in the system.

For the above points, some validation experiments were designed for the test system in remote wireless data transmission and related analysis.

The CM3160P GPRS Date Transfer Unit (DTU) wireless information transmission device developed by CaiMore Communication Technology Co., Ltd. in Xiamen is adopted in the program. This product provides online technical sustain and maintains data terminals permanently online and data link unblocked at any time to achieve the function of high-speed, stable and reliable Transmission Control Protocol/ User
Datagram Protocol (TCP/UDP) transparent data transmission[3].

\section{THE COMPARISONS OF GPRS WIRELESS COMMUNICATION SCHEMES}

DTU module based on wireless communication provides three remote data transmission schemes $(\mathrm{A}, \mathrm{B}, \mathrm{C})$, combined with the test system requirements some relevant confirmatory tests will be carried out. One of three schemes will be selected after comparing their advantages and disadvantages.

\section{A. DTU TRNS Mode Program}

Short Messaging Service (SMS) is accompanied with a digital mobile communication system resulting from a telecommunications service, which is a method that user can transfer messages through their mobile phones or other telecommunication terminals from point to point .

GPRS DTU wireless modules achieve transferring data by short messages which go through the network elements such as Global System for Mobile Communications/Code Division Multiple Access (GSM/CDMA) network, SMS center and so on. We electrify the DTU according to the hints of configuration software and set the CM3160P GPRS DTU working mode at TRNS. And then we conFigure the ID and the Subscriber Identity Module (SIM) card number of the DTU. After that, the DTU will start the SMS function automatically when turning power on.

During the confirmatory experiment, the data transfer between two modules as the form of short messages by the SIM card inside the DTU. Because every SIM card has a unique phone number as the identity of data sender and receiver, so there is no need to apply a new IP. However, each SMS message length cannot be more than 160 alphanumeric characters, which is subject to the mechanism of the signaling network communications system and is not suitable for transferring large amount data. At the same time, SMS message is transferred in a way of store-forward which is in a low speed and cannot transfer information in real time. In view of our system's requirement for large amount data transferring in real time, the DTU TRNS mode program was abandoned.

\section{B. DTU with APN Private Network Program}

Access Point Name (APN) is a private network using Virtual Private Network (VPN) technology for dedicated 
access services provided by mobile operators. It is to meet customers' special requirements for data transmission.

A program of using CM 3160P GPRS DTU with the SIM card which has APN private network business to achieve remote data wireless transmission from point to point was designed.

When the DTU with mobile APN private network is used to transfer data, all data go into the APN GPRS private network without going through the public network. So that it is possible to achieve transmission of large amounts of data in high speed, high frequency and high stability. While you can use the TCP transmission control protocol to ensure that no data is lost during transmission. Although advantages of this program on the data transfer are obvious, because the application cycle costs too much time, and the cost is high, which leads the program to be not suitable for laboratory validation experiments.

\section{DTU with Cloud Platform Program}

SaaS is Software-as-a-Service for short and rises in the 21 st century, which is a completely innovative software application model with the development of Internet technology and applications mature.

It is a mode of offering software service through the Internet. Application software vendors deploy applications on their servers. Customers can choose the service according to their actual needs and pay the vendor cost which depends on how many services and how long you use and access to services provided by the vendor via the Internet. The service providers will fully manage and maintain the software [1].

The cloud device management platform of CaiMore Communication Technology Co. in Xiamen adopts SaaS technology model. Its server of the cloud device management platform is a data center software which has a function of forwarding the data that sent by DTU to DTU client without any treatment. Different devices can communicate with each other and have the function of remote configuration.

The specific method of the design with SaaS cloud platform for the data center to achieve double-ended DTU remote wireless data transfer is as follows: The ordinary SIM card was used as the identification and CM3160P GPRS DTU was chosen to transfer data through cloud platform server-side. After configuring the DTUs, the remote data wireless transmission can be realized between two devices.

According to theoretical analysis and some comparisons of the above, it is known that the third data wireless transmission scheme has more obvious advantages than others. It cannot only meet the requirements that include large data transmission capacity, high frequency and short delay time of the test system, but also by the means of cloud platform provided by communications companies we don't need additional cost in the experiments. So we decided to choose the third option to test the system.

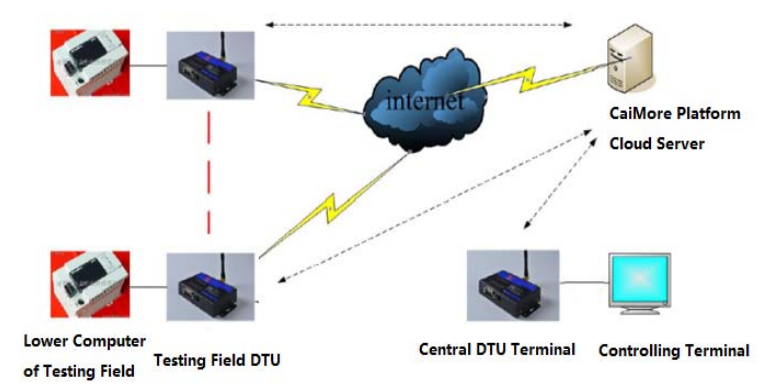

FIGURE I. THE WORKING PRINCIPLE OF CLOUD PLATFORM

\section{EXPERIMENTAL VERIFICATIONS OF THE DATA TRANSMISSION SYSTEM}

We constructed a program that can send and receive data on the software LabVIEW platform to validate the correctness and data transfer performance of the designed GPRS data transmission system. The double-end test system acquires the standard time information with Global Positioning System (GPS) timing service [4]. The head test equipment sends the current standard time information continuously at a fixed-rate (proximately one set of time information data per second) and the end test equipment receives the information that the head one sent and labels the time when the end test equipment received the time information. And then two sets of the time information data will be saved into the specified folder. The program will read the data and display them on the front panel of the program. The diagram of the time data is as Figure II describes.

Figure II shows 10 sets of time data which the end test equipment received. From these data we know that the head test equipment continuously sent a set of data with time information per second approximately and the end one received the data within one second without data loss. In this experiment, the head test equipment and the end one are both in Harbin city while the data cloud platform is set up in Xiamen city. The data transfer between the head test equipment in Harbin and the data processing center cloud platform in Xiamen, which spans a long distance. And that also increases the time of data transmission.

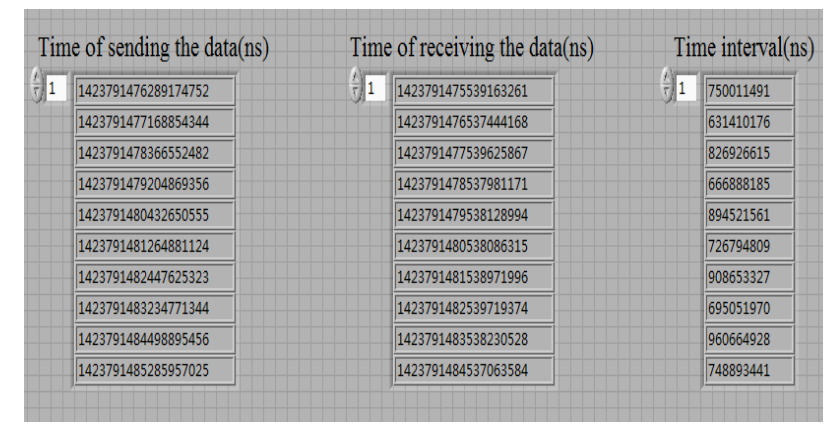

FIGURE II. THE DIAGRAM OF READING TIME PROGRAM ON THE FRONT PANEL 


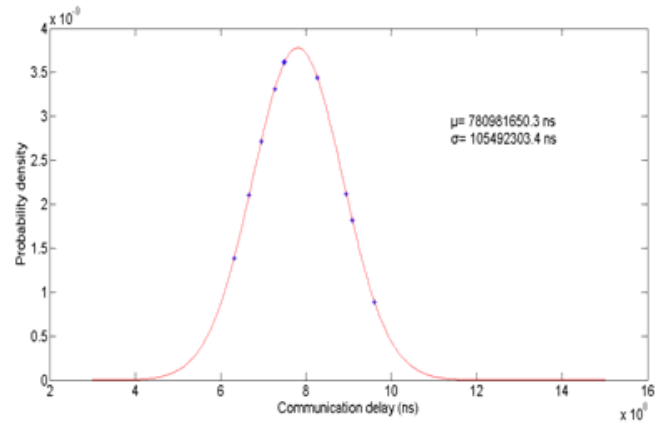

FIGURE III. THE NORMAL DISTRIBUTION OF COMMUNICATION DELAY

\section{ANALYSIS}

As Figure III shows, the data on communication delay between the head test device and the end device were statistically analyzed to obtain the normal distribution curve of communicating delay by maximum likelihood estimation method. It can be seen that the average of these data is $780981650.3 \mathrm{~ns}$ and the standard deviation is $105492303.4 \mathrm{~ns}$. The normal distribution can be used as a reference to direct the follow-up communication debugging.

A successful communication is defined as that the sampling order arrives at the end device from the head device on time, otherwise the communication fails.

There is a time margin $\left(\mathrm{t}_{\mathrm{m}}\right)$ among every data acquisition in order to make sure that the communication succeeds. According to the normal distribution curve, the table of $t_{m} v s$. communication success probability is as Table I shows.

TABLE I. TIME MARGIN $\left(t_{m}\right)$ VS. COMMUNICATION SUCCESS PROBABILITY (P)

\begin{tabular}{|c|c|}
\hline Time Margin $\left.\mathbf{( t}_{\mathbf{m}}\right)$ & Communication Success Probability (P) \\
\hline $0.78 \mathrm{~s}$ & $49.63 \%$ \\
\hline $0.80 \mathrm{~s}$ & $57.15 \%$ \\
\hline $0.85 \mathrm{~s}$ & $74.35 \%$ \\
\hline $0.90 \mathrm{~s}$ & $87.04 \%$ \\
\hline $0.95 \mathrm{~s}$ & $94.54 \%$ \\
\hline $1.00 \mathrm{~s}$ & $98.11 \%$ \\
\hline $1.02 \mathrm{~s}$ & $98.83 \%$ \\
\hline $1.05 \mathrm{~s}$ & $99.46 \%$ \\
\hline $1.10 \mathrm{~s}$ & $99.88 \%$ \\
\hline $1.50 \mathrm{~s}$ & $99.99 \%$ \\
\hline
\end{tabular}

It can be seen that the success probability goes up with the increase of time margin. When the time margin is $0.95 \mathrm{~s}$, the success probability is $94.54 \%$. While the margin is $1.00 \mathrm{~s}$, the probability is $98.11 \%$. But after then, the increase of the probability is not obvious when the margin continues going up.
So, the margin should be selected with the consideration of the whole system in order that the system can achieve an optimal working state.

When the time margin is set to be $1.00 \mathrm{~s}$, the communication mechanism is as follows: after the head device sent the sampling order, if the end device received the order beyond $1.00 \mathrm{~s}$, then the communication failed and the end device returned the failure information. And then the head device reset the order and resent it to the end device, and so forth until the communication succeeds. According to the Table I, it can be calculated that the failure probability of two communications in succession is $0.04 \%$, which can meet the need of the test system.

\section{CONCLUSIONS}

This article simply introduced the GPRS wireless transmission technology and several different schemes of wireless data transmission from point to point. And some relevant verification experiments were carried out. From the experimental results it can be seen that by using CM3160P GPRS DTU with the cloud server to realize long distance wireless data transmission from point to point is more suitable than others for our systems. And by the mathematical statistical analysis on the communication delay, finding the sampling interval time to meet the requirements of the system, which made preparations to instruct the follow-up communicating debugging.

\section{ACKNOWLEDGMENT}

Financial support obtained from the State Grid Electric Power Research Institute in Jiangsu Province in China is greatly acknowledged.

\section{REFERENCES}

[1] Binhua Shen, "Investigation of Localized Production of HV Insulation Compound". Electric Wire and Cable, No.1, Feb. 2010.

[2] Xinlao Wei, Bo Zhu, Bing Pang, Song Wang, Ruihai Li, "On-line Insulation Monitoring Method for Long Distance Three-Phase Power Cable," Proceedings of the Chinese Electrical Engineering Science, vol.35 No.8, Apr. 20, 2015.

[3] Bo Zhang, Ji Gao, "Trusted autonomic service cooperation multi-tenant tenancy model," Computer Engineering and Applications, vol.47, pp. 67-71, Nov. 2011.

[4] Bo Hao, Jun Yue, Changyong Yin, "Research of high precision time signal based on synchronized phase measurement," Journal of Shenyang Institute of Engineering(Natural Science), vol.3, no.3, pp.276-278, Jul.2007. 